\title{
La construcción de los buenos vivires; entre los márgenes y tensiones ontológicas
}

\author{
Juan Jaime Loera González \\ Pontificia Universidad Católica de Chile.Santiago, Chile. \\ Email: Jloera@uc.cl
}

\begin{abstract}
Resumen: El presente artículo pretende entablar una discusión teórica planteando la noción de los márgenes como espacio-proceso donde se articulan proyectos políticos relacionados al Buen Vivir. Dicha propuesta hace referencia a la fluctuación que ejercen pueblos con otras historias, incluidos los pueblos indígenas de Latinoamérica, para moverse dentro del Estado, el mercado y la sociedad dominante, y a la vez de retraerse y disentir de élapelando a sus diferencias ontológicas. Argumento que dicha fluctuación, representa fuerzas complementarias más que opuestas, que expresan el derecho de vivir diferente a la sociedad dominante, y al mismo tiempo, a la necesidad de pertenecer a ella. La tensión creada entre ambas fuerzas es fundamental para entender elBuen Vivir en el contexto políticoLatinoamericano contemporáneo.La propuesta de los márgenes, por ende, abre la posibilidad de un diálogo de saberes, pero también ofrece una línea de escape ante la colonialidad del proyecto de la Euromodernidad.
\end{abstract} co, Chile.

Palabras clave: Buen vivir,márgenes, ontologías; pueblos indígenas, Méxi-

\section{The building of good living; between the margins and ontological tensions}

\begin{abstract}
This paper aims to propose a theoretical discussion concerning the notion of the margins as a space-process where political projects related to BuenVivir are articulated. This proposal refers to the fluctuation experienced bypeople with different histories than that of modernity,includingindigenous peopleof Latin-America, in order to move within and outside the state, markets and dominant society. It also includes theirability to withdraw and dissent from the state appealing to their ontological differences. I argue that such fluctuation can be understood as complementary rather than as opposing forces, expressing the right to live differentlyin regards the dominant society, and at the same time, the need to belong to it. The tension created between this two forces is essential to understand the BuenVivir in the context of contemporary Latin American politics. The proposal of the margins thus opens the possibility of a dialogue of knowledge's, but also offers an escape to the colonialist project of Euro-modernity.
\end{abstract} Chile.

Key words: BuenVivir, margins, ontologies, indigenous people, Mexico; 


\section{A construção dos bons viveres; entre as margens e as tensões ontológicas}

Resumo: Este artigo tem como objetivo estabelecer uma discussão teórica, argumentando a noção das margens como espaço-processo onde se articulam os projetos políticos relacionados ao Bom Viver. Esta proposta refere-se à flutuação exercida pelos povos com outras histórias, incluidos os povos indígenas da América Latina, no seu movimento dentro do Estado, do mercado e da sociedade dominante, e por sua vez de retrairse e discordar dele apelando para suas diferenças ontológicas. Argumento que essa flutuação representa forças complementar mais que forças opostas, expressando o direito de viver diferente da sociedade dominante, e, ao mesmo tempo, a necessidade de pertencer a ela. A tensão criada entre as duas forças é essencial para entender o Bom Viver no contexto político latino-americano contemporâneo. A proposta das margens, assim, abre a possibilidade de um diálogo de saberes, mas também oferece uma via de escape antea colonialidade do projeto da Euro-modernidade. co; Chile

Palavras-chave: Bom Viver; margens; ontologias; povos indígenas; Méxi-

\section{Introducción $^{1}$}

A la luz de recientes movilizaciones de pueblos originarios y grupos marginalizados que han surgido en Latinoamérica, ha emergido un creciente interés por considerar las diferencias ontológicas como perspectiva clave para explicar desde una mirada más amplia demandas políticas de reconocimiento de derechos colectivos o diferenciados.El interés por los modos de entender el mundo de manera alterna al de la Euro-modernidad ${ }^{2}$ y su proyecto neoliberal, ha dado pie a revisitar sus supuestos fundacionales, tales como el dualismo naturaleza/sociedad; la prioridad del crecimiento económico y extractivismo; la jerarquización de saberes locales, incluso la tensión de concebir la cultura como estructura simbólica para pensar en ella en su dimensión política como diferencia radical (Escobar 2012, Blaser 2009, 2010).

En definitiva, lo que está en el horizonte de estas críticas a lo dominante y a la búsqueda de lo alterno es la exploración de nuevas y radicales concepciones de aquello que todavía llamamos desarrollo. Para ello, se requiere de innovadoras vías para entretejer políticas y saberes que estén encaminadas a la construcción de puentes comunicativos, a un dialogo de saberes que trascienda los graves dificultades y límites impuestos por la modernidad y al mismo tiempo que logren cimentar espacios de intercambio y construcción.

El presente artículo tiene el objetivo de explorar tres puntos específicos. Primeramente, exploro la necesidad de reconocer la pluralidad de significados, discursos y proyectos relacionados al Buen Vivir que están en continua construcción y surgen en diversidad de contextos, y por ende, no 
se debe limitarse a entenderlo como un solo proyecto terminado. Este punto se abordara en la segunda sección.

Posteriormente, el segundo punto presento la propuesta analítica de los márgenes como espacio-proceso para analizar los proyectos y propuestas en torno al Buen Vivir. Argumentoque dichos proyectos políticos de los Buenos Vivires,surgidos desde los pueblos con historias diferentes a la modernidad y que enarbolan formas alternas de convivencia y desarrollo, son generalmente analizados y concebidos al margen de un solo centro comúnmente siendo este centro la figura del Estado- cuando se deben visualizar lo que esta fuera del alcance del Estado mismo. Es decir, dichos proyectos están también dentro de la fuerza gravitacional de otros centros, por ejemplo, las comunidades política-ontológicas indígenas. Por ende, postulo que los pueblos con otras historias-incluyendo los indígenas en Latinoamérica- se han adaptado y articulado en el espacio de los márgenes, a veces identificándose dentro del entramado político del Estado y de la Euro-modernidad y en ocasiones alejándose y posicionándose fuera del mismo. Para ejemplificar este enfoque policéntrico, menciono el caso del pueblo Rarámuri del Norte de México y el pueblo Mapuche de Chile y sus formas de articulación política fluctuante, en diferentes grados y ámbitos. En este sentido, los proyectos del Buen Vivir se posicionan en la tensión entre dos fuerzas; ocupando espacios estatales al demandar derechos igualitarios y ciudadanía diferenciada, y en ocasiones ocupando espacios fuera de los principios del mercado y el estadopara fortificar su capacidad autogestiva como poseedores de proyectos políticos alternos. Es en este balance de fuerzas, en esa tensión no ausente de conflictos y contradicciones, donde hace sentido conceptualizar los proyectos del Buen Vivir.

Finalmente, el tercer punto desarrollado en la cuarta sección exploro como esta fluctuación entre ambas fuerzas hace eco en algunos planteamientos dentro de la tradición de los estudios del decolonialidad y del giro ontológico. Ello se planteará a través de discutir la pertinencia de abordar una necesidad de dialogo de saberes mediante a la propuesta teórica de los márgenes. En este punto propongo incorporar un sentido procesal en tres momentos; aceptar, en un primer momento, que un proyecto político que pretende romper con tantas supuestos de la modernidad debe ser radical en su pretensión decolonial, para pasar a un segundo momento donde habiendo definido su radicalidad, se pueda generar un dialogo de saberes entre posturas ontológicas que reconozca el mismo carácter plural y holístico del Buen Vivir. Como tercer momento, incorporo la necesidad de tener mecanismos dentro de los proyectos políticos alternos de seleccionar conocimientos y prácticas de la Euro-modernidad que se quieran incorporar, además de tener la capacidad de retraerse y disentir del modelo político imperante para poder generar un dialogo en igualdad de condiciones y evitar o por lo menos disminuir las relaciones asimétricas persistentes de la modernidad. 


\section{Buenos Vivires}

Para empezar a hablar del Buen Vivir, creo que hay que comenzar por precisar que hay Buenos vivires, en plural, no en singular. No hay una sola definición del Buen Vivir completa ni acabada. De hecho, para Eduardo Gudynas (2011), una de las características del Buen Vivir es que es un proyecto en construcción, cuya idea se refiere más a una plataforma que a un concepto acabado y preciso. No es lo mismo el Buen Vivir del cual habla Evo Morales como expresión del proyecto de descolonización, al Buen Vivir de SimónYampara (2011), quien habla de la importancia de la complementariedad de saberes y conocimientos occidental e indígena, o el Buen Vivir de Rafael Correa, lo que él llama socialismo del siglo XXI basado en continuar con un extractivismo pero que tiene el objetivo de generar y distribuir riqueza para impulsar políticas publicas progresistas; al buen vivir de su compatriota Alberto Acosta (2008, 2011), que tiene una fuerte presencia del enfoque de derechos, incluidos los derechos de la naturaleza; o finalmente, como lo entiende Quijano (2012), para quien debe ser un proyecto completamente diferente y radical en su pretensión decolonial. Existe por tanto una gran diversidad de ideas, proyectos y discursos asociados al Buen Vivir.

Los proyectos sobre el Buen Vivir a los cuales me referiré por lo tanto, es paralelo a lo propuesto por Gudynas (2011) en el sentido de concebir un plataforma desde la cual se han articulado tres puntos fundamentales: el primero es una crítica al modelo imperante de desarrollo neoliberal orientado a la ganancia. El segundo es una reivindicación de sabidurías y ontologías históricamente excluidas entre ellas las de los pueblos indígenas. En este respecto, el Buen Vivir se nutre fundamentalmente en las raíces andinas del SumakKawsay (Quechua) Suma Kamaña (Aymara). Y por último, el proyecto de Buen Vivir del cual me refiero en esta ocasión implica también considerar un cambio de paradigma antropocéntrico imperante en los modelos de desarrollo occidental para apostar en cambio por un paradigma biocéntrico que implica reconocer una forma diferente de vivir y concebir la naturaleza y realidad(es). Por ende, los proyectos ostentan un fuerte sentido político, en el sentido de que reivindican un proyecto en construcción permanente con un fuerte sentido local y contextualmente especifico de cada pueblo, y por ende cada pueblo debe definirlo, sistematizarlo y construirlo. No puede haber recetas fijas, solo aprendizajes y adaptaciones, y al mismo tiempo capacidad de seleccionaraquellos conocimientos que puedan ser útiles, y contar mecanismos de asentir y disentir.

Esta plataforma del Buen Vivir implica asumir una alternativa al paradigma de la Euro-modernidad al considerar articulacionesde ensamblajes ontológicos alternos.Ello involucra, frecuentemente, tensiones y disputas de saberes y espacios vividos producto de la exclusión, invisibilización y/ o reduccionismo de sistemas de conocimiento de pueblos con otras historias por parte del proyecto dominante de la modernidad.

$\mathrm{Al}$ respecto, Mario Blaser habla de la ontología política como "las 
negociaciones que se dan dentro de un campo de poder en el proceso de gestación de las entidades que conforman un determinado mundo u ontología (...), así como en los conflictos que se generan cuando estos dos mundos u ontologías tratan de sostener su propia existencia al mismo tiempo que interactúan y se mezclan con otros diferentes” (Blaser 2009; 83). En este tenor es que planteo la propuesta analítica de considerar los Buenos Viviresen el espacio de los márgenes como lugar-proceso necesario donde puedan coexistir saberes mediante una constante articulación de diferencias ontológicas. Es decir, los márgenes son un lugar de lucha entre regímenes de verdad, de realidades contestadas y encuentros no ausentes entre tensiones y controversias entre distintos proyectos políticos. ${ }^{2}$ Pero también esta concepción de los márgenes implica potencialidades, y por lo tanto, oportunidades y desafíos. Las oportunidades son la posibilidad de un dialogo de saberes y prácticas anti-hegemónico alejado de centros de dominación ontológica, modernos o no modernos. Los desafíos se refieren a las provocaciones sublimes del proyecto de la modernidad de simplemente incluir 'al otro' reduciendo su diferencia ontológica a la comprensión moderna dominante. Caer de nuevo en máscaras y fachadas que substituyen discursos pero donde la correlación de fuerzas se mantiene.

\section{Los Buenos Vivires situados entre los márgenes ontológicos}

Cuando hablo de articulación de ensamblajes ontológicos alternos pienso en la habilidad que muestran los Rarámuri en México, los Mapuche en Chile, y otros pueblos de moverse entre mundos. En esos encuentros, muchas veces vividos enuna correlación de fuerzas históricamente disparejas, existen conflictos, olvidos, discriminación, y silencios, pero también existen espacios de autonomía donde se guardan celosamente conocimientos, rituales y prácticas en donde se gestan proyectos políticamente alternos, no solo culturalmente distintos. En palabras de SimónYampara (2011), las culturas indígenas han sabido sobrevivir y convivir con los diversos, no solo con la diversidad, sino con los diversos mundos. En este sentido, argumento que estos ires y venires entre los diversos mundos implican algo más que estrategias de subsistencia, sino que permiten visualizar encuentros comunicativos que operan en ámbitos de conocimientos y prácticas entre diferentes ontologías.

Las fronteras de estos mundos generan espacios donde se expresan, articulan y reconfiguran sentidos y significados de ciudadanía, derechos, proyectos políticos y sentidos de vida inherentes a la realidad y condición humana, sentidos de bienestar. Los márgenes, son espacios en donde se confrontan y definen nuevas epistemologías y proyectos ontológicos. Son espacios de contención y lucha, de tecnologías de poder, muchas veces de opresión pero también de oportunidad política y encuentros. En este sentido, los márgenes son espacios de potencialidad, es la zona gris que es y no es al mismo tiempo, lo que puede llegar a ser o no. La 
cuestión, como bien nos recuerda Povinelli (2011), es un asunto de distribución de intensidad de potencialidad. Hay lugares-proceso que poseen más potencialidad transformadora que otros.

Amplios sectores de los pueblos originarios, los pueblos que tienen otras historias que se distancian de los proyectos civilizatorios dominantes, se encuentran en los márgenes. No solo como comúnmente se les asocia, en la periferia de los Estados o de los mercados globales, sino también dichos pueblos, o sectores de ellos, se posicionan en los márgenes ontológicos. Los márgenes a los cuales me refiero, por ende, no implican marginalidad, ni económica ni geo-espacial, sino una locación estratégica de interacción entre dos o varios mundos o realidades ontológicas distintas.

Por una parte los pueblos con otras historias se encuentran en los márgenes de una red de relaciones políticas asimétricamente estructuradas que se orientan hacia el libre mercado y la mercantilización de la cotidianidad. Y, por otro, ostentan ciertos espacios auto-definidos donde (re)producen prácticas políticas y culturales definiendo y renovando su identidad étnica. Aunque tienen un pie en ambos campos, no ocupan completamente uno o el otro. Esta condición de estar en los márgenes representaser económica y socialmente vulnerables debido a la falta de un adecuado reconocimiento a lo radicalmente alternodesde la configuración de la sociedad dominantey de los derechos socio-culturales y económicos por el gobierno nacional. Sin embargo, dicha condición en los márgenes también permite gestionar decisiones estratégicas a fin de negociar los términos generales de las relaciones con el predominio de los estados - y para gestionar su papel como pueblos y proyectos diferenciados con el Estado y la sociedad nacional. En definitiva, los márgenes permiten adoptar un enfoque estratégico para hacer frente a las vulnerabilidades económicas como resultado de procesos de exclusión, así como también para beneficiarse de las oportunidades que su posición de vivir en los márgenes en términos de reforzar su capacidad autogestiva y políticamente diferenciada.

Por consiguiente, propongo que los discursos políticos de los Buenos vivires, se deben de operacionalizar en el fluctuante contexto de los márgenes; esto significa que fluctúan entre la tensión de tener el derecho a vivir de otra manera a la dominante y la necesidad de ser parte de la sociedad en general(Loera Gonzalez 2014a; Loera Gonzalez 2014b). La idea del Buen Vivir consiste en la búsqueda de un equilibrio entre un estado físico, social y espiritual en un caminar complementario entre varios mundos, y potencialmente entre varias ontologías. Por lo tanto, los márgenes más que crear híbridos ontológicos, son espacios de continua articulación de diferencias y similitudes, espacios en donde se puede convivir con traductores y controversias, que permitan la existencia de múltiples mundos, sin que ellas sean reducidas constantemente a diferencias o similitudes culturales, sino entenderlos como proyectos y demandas en su dimensión político/ontológico. ${ }^{4}$

En otras palabras, la posición en los márgenes y del Buen Vivir re- 
quiere tener un enfoque que vaya más allá del Estado. Debe ser multicentrada en sus relaciones dinámicas entre el Estado y los pueblos con otras historias, incluidos los indígenas, entre el núcleo de cada una de las comunidades políticas y culturales. Esto implica tener una nueva cartografía de cómo considerar los márgenes. Con demasiada frecuencia, estar en los márgenes sólo se entiende en relación a un centro único, siendo el Estado el más común ejemplo de centro estructurante. El Estado como la única comunidad política que tiene el monopolio legítimo de ordenar las relaciones humanas, es quien organiza, otorga y niega derechos, genera y etiqueta sujetos, sin embargo, en entornos multi-ontológicos, donde los pueblos indígenas están involucrados, sus propios proyectos políticos y formas distintivos son importantes. Si tenemos en cuenta la literatura, por ejemplo, sobre la etnografía del Estado -Das y Poole (2004) por mencionar un ejemplo- definen muy bien al Estado, como aquel conjunto de prácticas y espacios en donde el control y regulación disciplinante de la vida se hace evidente; fronteras, puestos de control, instituciones gubernamentales, prácticas de salud, etc. Sin embargo, en dicho material los márgenes son entendidos como la ausencia o disminución de la influencia del Estado, sin considerar aquello que existe más allá del Estado. En el enfoque policéntrico que estoy proponiendo, los márgenes no sólo se refieren a la ausencia o disminución de la influencia del Estado, sino también implica la existencia de otra comunidad política, digamos el centro de la comunidad política/ontológica Rarámuri o Mapuche. En ese sentido, otros etnógrafos del Estado apuntan en la misma dirección. Clastres (1978), por ejemplo, da cuenta de un Estado en forma potencial presente en todas las sociedades pero que estas tendrían la capacidad de generar mecanismos paraevitar su constitución. Incluso aun va más lejos al hablar que el Estado es la señal acabada de la división de la sociedad ya que es el ente que separa a los sujetos que ostentan el poder y aquellos que obedecen. En el mismo tenor, vale la pena mencionar el trabajo de Prada (2008) que estudia la realidad política boliviana actual a partir de una concepción anti-estatal del Ayllu andino.

Con esto, no argumento que exista una naturaleza anti-Estado en las sociedades indígenas, ni que las sociedades sin Estado sean un reclamo actual de las demandas políticas indígenas. Al contrario, creo que uno de los temas predilectos de la antropología del Estado ha sido mostrar lo persuasivo y a veces necesarioque es contar con un Estado eficaz en las relaciones con los pueblos indígenas. Sin embargo, como bien dice Quijano, las discusiones y debates sobre el desarrollo en América Latina, se han planteado en torno a la función del Estado debido a la hegemonía del paradigma del Eurocentrismo (2012:47). Considerando que otros paradigmas y proyectos políticos que han estado marginados y excluidos históricamente, se vuelve imperativo considerar la posibilidad de gestionar otros contratos sociales. La discusión no es si debe o no existir el Estado, sino más bien lo crucial es preguntarse qué tipo de naturaleza deberá de tener, qué constitución y con qué relaciones políticas se formará, y para ello se requiere reconocer que existen proyectos políticos alternos que se ubican fuera del Estado. 


\section{Buen Vivir como balance entre dos fuerzas: ¿Dentro o Fuera del Estado?}

La posición política donde se ubica el proyecto político del Buen Vivir en el entramado global actual de la descolonialidad es una discusión fundamental. Nancy Postero (2006) al analizar el movimiento indígena en Bolivia, así como Sarah Radcliffe (2012) al examinar el desarrollo en la era post-neoliberal en Ecuador, coinciden al preguntarse sobre si el Estado puede ser el locus de la tan deseada descolonización, proceso tanambicioso y transformador propuesto con la llegada de los gobiernos progresistas de Evo Morales y Rafael Correa respectivamente, y que se enmarcan con la creación de lasnuevasconstitucionesinspiradas en ideas sobre el Sumak Kawsay y del Suma Qamaña. Pareciera que la descolonización tal como la plantean ambos Estados tiene sus limitaciones. En cierta forma, la experiencia que ha surgido en ambos países pareciera imponer una forma en concreto de ser indígena. Una forma que si bien rompe simbólicamente con elementos Europeos y occidentales, éstos son sustituidos por una imagen homogénea de lo indígena al sobrerepresentary esencializar aspectos. También hay que reconocer que si bien la descolonización en Bolivia ha sido hasta ahora mayormente discursiva, ésta ha transformado el escenario de referencias históricas colonialesalterando las políticas públicasy estructuras simbólicas que organizan la sociedad Boliviana. Otros autores, entre ellos Acosta $(2008,2010)$ y (Acosta y Gudynas,2011) han ya analizado con detallelas implicaciones de las nuevas constituciones de carácter progresistas de Bolivia y Ecuador. Sin embargo, dicho ejemplo, abre la puerta para discutir posibilidadesde procesospara construir los diferentes proyectos del Buen Viviry a través de la participación de quienes. El caso de ambos países andinos indica que el Estadoha jugado un papel dominante en la orquestación de dicho proceso descolonizador y de implementar políticas públicas que responden a lo que el mismo Estado define como Buen Vivir.

En este sentido, argumento que un proyecto tan holístico como el del Buen Vivir debe surgir desdela participación plural de diversos actores en un proceso articuladoy en una multiplicidad de ámbitos y espacios, tanto dentro como fuera del Estado.

La base etnográfica de dicho argumento es visible al analizar el casode algunos pueblos indígenas, como por ejemplo, el pueblo Rarámuri (Loera Gonzalez, 2013). El pueblo Rarámuri basa la búsquedade un cierto tipo de Buen Viviren el balance entre dos fuerzas: a) el derecho de vivir de manera diferente a la propuesta dominante con su propio proyecto político, ejerciendo control y poder sobre sus instituciones políticas, religiosas, sociales y manteniendo prácticas y mecanismos de toma de decisión y organización autónomas; y, b) la necesidad/exigencia de ser parte activa de la comunidad política delEstados y la sociedad mayoritaria con el objetivo de articularse en igualdad de condiciones como sujetos y ciudadanos dentro del imperante proyecto político económico neoliberal y globalizante. 
Por ejemplo, como parte del derecho de vivir de forma diferente, buena parte del pueblo Rarámurimantiene una fuerte conexión con la agricultura de autoconsumo como forma de sostenimiento,de existencia política y cultural diferenciada a la sociedad dominante. Dentro de un discurso étnico altamente identificable entre los Rarámuri, la expresión del caminar bien y caminar por el camino correctoestá constituida por el imaginario ideal de cómo uno debe de comportarse y actuar en la vida para tener Buen Vivir, y que importantemente se relaciona convivir de su agricultura. Por una parte, la agricultura de autoconsumo se relaciona a la conexión holística con la tierra, el mantener control territorial, las prácticas que sostiene redes de cooperación y solidaridad, el complejo acto ritual y religioso entrevecinos, familiares,entidades espirituales y ancestros, además de un mayorcontrol del modo de sostenimiento y la seguridad alimentaria. Es decir, el cultivo de autoconsumo implica el compartir el entorno con entidades humanas y nohumanas de manera intensa donde el sentirse parte de una comunidades más importante que la ganancia económica individual que supondría depender derelaciones de mercado a través de la comercialización de fuerza de trabajo o la comoditización de la vida cotidiana.

Es así que los Rarámuri procuran siempre tener una conexión con su territorio expresando su derecho a vivir de manera diferente a la del proyecto occidental y sin una intervención directa del Estado.

De manera conversa, el Buen Vivir para los Rarámuri no se limita a mantener prácticas autóctonas de autoconsumo, sino también implica la necesidad de verse como parte constitutiva de un entramado de relaciones sociales, económicas y políticas con el Estado y la sociedad dominante. Estas relaciones toman forma en la búsqueda de actividades generadoras de ingresos que proporcione seguridad monetaria en tiempos de precariedad y escasez, y en la exigencia de tener los mismos derechos y beneficios ciudadanos que el resto de la sociedad dominante; acceso a servicios básicos, beneficios de programas de protección social o bien la incorporación en el mercado laboral de manera parcial o semi-permanente en el campo o las ciudades. Expresiones de esta necesidad/exigencia incluyendemandas legales y luchas de defensa territorial en conflictos medioambientales o de reconocimiento propiedad y límites, basados en los derechos reconocidos en instrumentos legales e instancias jurídicas necesarias para su solución. Crucialmente, esta necesidad/exigencia de vincularse con el Estado y sociedad dominante requiereacontecer en igualdad de circunstancias sin representar las usuales formas de exclusión y discriminación que constituyen de relaciones de asimetrías sociales y económicas. Esto, por supuesto, implica para los Estados tener un marco legal con las herramientas apropiadas que favorezcan una participación activa en los sujetos y colectividades portadores de derechos, pero que también reconozca ciudadanías diferenciadas (Young, 1990) que les proporcione posibilidad de distinción del resto de la sociedad. Y aún más, implica la posibilidad de disentir del contrato social imperante. Esto tiene que ver con lo que Nozick (1974) denomina derecho negativo, en oposición al derecho positivo. El primero se refiere a los derechos que el Estado tiene de garantizar al individuo mientras que el 
negativo hace referencia al derecho de no interferencia. En efecto, implica contar con restricciones indirectas donde el Estado debe vigilar que por ninguna instancia, se vulnere la capacidad de disentir y retraerse del Estado, tal como señalarémás adelante.

En el mismo tenor, Elisa Loncon (2014), identifica la necesidad de descolonizar lingüísticamente las políticas sociales, políticas y económicas de Chile, para buscar reconocimiento a la existencia de otras formas de conocimiento indígenas.Formas deconocimiento que en la cultura mapuche tiene por objetivo alcanzar el kvmemogen, o Buen Vivir en Mapuzungun, e implica la interrelación de diversas prácticas, principios, valores, procesos cognitivos, vinculados entre sí. Discutiendo sobre la descolonización lingüística en Chile,Lonconmenciona que puede ser la ventana que abra "el sistema escolar Chileno a las lenguas y culturas indígenas, como dispositivos de descolonización que permitan fundar una nueva sociedad (...) que reconozca y se nutra de los cimientos originarios, principalmente de sus lenguas, conocimientos y valores”(2014). Estas posturas expresan un deseo de complementariedad y de generar un nuevo pacto fundacional con la sociedad dominante que reconozca epistemologías y saberes diferentes.

Adicionalmente, proyectos políticos Mapuche, como el elaborado por la Confederación Mapuche de Neuquén, relacionan la libre determinación a la identidad y las normas del kumefelen, o estar bien en Mapuzungun, en el sentido de que la autonomía se busca a través de vías jurídicas apelando a la Declaración de las Naciones Unidas sobre Derechos de los Pueblos Indígenas, así como otros instrumentos internacionales, nacionales y municipales que otorgan el derecho de los pueblos a la libre determinación, en virtuddel cual éstos determinan libremente su condición política y persiguen libremente su desarrolloeconómico, social y cultural. En ese contexto, los Estados son los encargados de garantizar el ejercicio a la libre determinación, a la autonomía o al auto gobierno en las cuestiones relacionadas con sus asuntos internos así como de disponer de los medios para ejercer tal ejercicio(Confederación Mapuce de Neuquén, 2010). Por lo tanto no se trata de crear un Estado dentro de otro Estado, sino de que salvaguardey reconozca derechos. Es decir, estos proyectos políticos alternos que siguen pueblos indígenas, buscan construir capacidad de autogestión de sus recursos sociales, culturales, naturales y espirituales y, a través de ello, construir una nueva relación con el Estado diferenciándose, hasta cierto grado alejándose -mas no saliéndose del todo- de él.

Por lo tanto, el Buen Vivir es buscado en la fluctuación de dos fuerzas, algunas veces inclinándose a un lado, en otras enfocando al otro, o en un amplio espectro que combina ambas en diferentes grados y dimensiones. Parece que la idea del Buen Vivir como proyecto político consiste en precisamente mantener un balance armonioso y efectivo entre ambas tensiones. Es por ello que el Buen Vivir cobra sentido en el espacio entre los márgenes. Desde una perspectiva existe una clara dimensión de rescate y preservación de sabidurías, conocimientos y prácticas distintivas de los pueblos, y desde otro ángulo, una dimensión de relaciones necesarias con 
Estados y mercados. Si consideramos numerosos grupos de pueblos indígenas en Latinoamérica, observaremos que su forma de vida sigue el mismo camino; balanceándose entre prácticas gobernadas y controladas por sus instituciones y, al mismo tiempo, relacionándose con Estados y mercados tratando de sostener relaciones en igualdad de condiciones con otros actores. En cierta forma, esta economía mixta se aleja de la lógica monoproductiva para apostar por diferentes alternativas, seguir la línea estratégica de la diversificación productiva y la búsqueda de participación en espacios socio-políticos.Ello implica adaptación para hacer frente a vulnerabilidades y mantener su identidad como pueblo indígena.

El potencial de tener el derecho a existir fuera del Estado, de vivir de manera diferente es múltiple. Considero que las formas de luchas contemporáneas que buscan la construcción de proyectos autónomos, como por ejemplo las comunidades Zapatistas, se basan en la organización propia de sus bases sociales y políticas. Apuestan por ende a fortalecer y ejercer de manera propia el control de prácticas e instituciones sociales, educativas, políticas y culturales. En ocasiones la estrategia es compartida con el Estado aprovechando aquellas herramientas legales, programas y espacios de verdadera participación colectiva. En otras ocasiones la estrategia es jugar fuera de la cancha del Estado, cambiar el juego hacia la cancha comunitaria apostando más a la organización propia y la construcción de la autogestión. Esto implica cambiar la conversación desde el terreno político usual hacia otro terreno de consenso y organización local y volver la mirada hacia las comunidades de abajo. Al jugar fuera del Estado, se está anulando la existencia de aquellas instancias dominantes y relaciones de opresión tejidas desde estructuras más amplias. Hacerlo, implica una orientación de no asumir una posición "anti” y adoptar una posición más hacia "no cómplice”; no cómplice de un orden de gobernanza que robustece poderes, no cómplice de monopolios económicos, políticos y ontológicos. Al no ser cómplice de ello, se deja de otorgarle poder.

Estar afuera de la influencia del Estado es una forma de lucha que cobra mayor importancia actualmente dentro de los movimientos altermundistas, inspirada, entre otras fuentes, por las nuevas lecturas del anarquismo. Sin embargo, no hay que olvidar la otra parte de la ecuación; la necesidad/exigencia de tener relaciones equitativas con los amplios sectores de las naciones, demandar reconocimiento como sujetos de derecho jurídico colectivo e individual, trato igualitario y no discriminación en las actividades productivas de la nación. Dicha exigencia es una sentida necesidad de los pueblos indígenas de Latinoamérica, siendo tal vez la que articula las relaciones políticas más visibles en la actualidad. Por ello, una propuesta de acción, de búsqueda de Buen Vivir, se encuentra en tensión entre ambas fuerzas. En un espacio en donde ambos caminos, o mejor dicho un amplio abanico de opciones fluctuantes entre estas dos fuerzas, se estén constantemente celebrando.

A continuación argumentaré que esta fluctuación entre ambas fuerzas hace eco a lo planteado por los autores del giro ontológico (Blaser, 2009, 
2010, 2012)y del decolonialismo, entre ellos Quijano (2012) yMignolo(2000) en relación a las luchas de grupos minoritarios por su reconocimiento y diferenciación radical como poseedores de proyectos políticos alternativos a la Euro-modernidad.

\section{Buen Vivir como dialogo de saberes; la opción de los márgenes}

LaEuro-modernidadtiene como principio la tendencia de reducir otros saberes a meras expresiones y creencias culturales jeraquizables, es decir sugiere la invisibilización y exclusión de otros conocimientos, otras lógicas y mecánicas de representación que surgen de ensamblajes ontológicos alternos, de pueblos con otras historias. La tendencia de invisibilización/ exclusión hace que las diferencias del pueblo Rarámuri o Mapuche con la sociedad dominante se entiendan de carácter cultural, y no político en el sentido de que un sistema de pensamiento persiste en mantener al otro en una posición de dominación colonial. Por lo tanto, entender como los pueblos con otras historias fluctúan entre el derecho de mantener control autogestivosobre sus instituciones y la necesidad/exigencia de mantener relaciones con esferas gubernamentales dentro del proyecto neoliberal/ moderno implica aportar luces no solo en cómo se puede resistir y luchar políticamente tanto dentro como fuera del Estado, sino también implica en sí mismo la necesidad de plantear un dialogo de saberes en un escenario donde el reconocimiento de otras ontologías y la misma diversidad humana y no-humana está en juego.

En definitiva, esta condición de estar en los márgenes representael reto para el Buen Vivir de fungircomo un espacio de diálogo político y ontológico. En este sentido, existen varias posturas encontradas sobre la naturaleza del Buen Vivir en su relación con el Estado y la modernidad. Por un lado, Aníbal Quijano (2012) establece que el Buen Vivir requiere plantearse como prácticas sociales radicalmente alternas en sí mismas a la colonialidad del poder y a la colonialidad/modernidad/eurocentrada.Para él, toda elaboración sobre el Buen Vivir se tiene que ubicar en el contexto de la descolonialidad del poder y la irrupción de nuevas resistencias altermundistas donde los reclamos no son solo sobre la pobreza y desigualdad, sino sobre las condiciones mismas de sobrevivencia en la tierra. Comparto completamente con esa premisa básica en donde el proyecto político del Buen Vivir se constituya en el otro radical pues para que el Buen Vivir se establezca realmente como arquitectura alternativa, tiene que diferenciarse radicalmente de aquellos supuestos modernos que invisibilizan y jerarquizan conocimientos y mundos con otra construcción que se encuentre completamente afuera del edificio ontológico moderno, como por ejemplo, para remplazar el paradigma antropocéntrico por el biocentrismo en cuanto a la sustentabilidad. Tal como lo proponen Quijano y otros autores de la decolonialidad, el Buen Vivir tiene que plantear un horizonte epistémico y ontológico totalmente radical al proyecto del desarrollo de la modernidad 
dominante actualmente. Estoy de acuerdo con ese argumento, sin embargo, yo argumento que se puede plantear un punto de equilibrio. Para ello habrá que incorporar un elemento de temporalidad.

El Buen Vivir como proyecto totalmente radical a la modernidad, es tan solo el primer paso; tal diferenciación radical es útil para darle postura y carácter a la propuesta del Buen Vivir (de los múltiples Buenos Vivires que se estén reivindicando en un contexto específico). Una vez que se posicione el discurso y la propuesta del Buen Vivir como proyecto alterno al desarrollo, entonces podrá establecer las condiciones de diálogo y negociación con los otros modelos culturales y ontológicos dominantes.En otras palabras, la esencia radical dentro del proyecto del Buen Vivires la condición necesaria que permite celebrar un diálogo donde las posiciones sean claras y poder llegar a una posición a la conversación en un marco plural. Con ello, apuesto a que pueda existir una definiciónholística del Buen Vivir: un Buen Vivir que se nutra del diálogo de fronteras, y que se nutra de la fluctuación de los márgenes políticos y ontológicos y que permita la articulación de epistemologías relajadas y supuestos ontológicos excluidos sin relaciones de subordinación ni hegemonías.

En este mismo sentido, Vanhulst\&Beling (2013; 2013a) establecen de manera clara que si el Buen Vivir va a participar en el debate global sobre el desarrollo debe asumir una posición plural y no desechar la modernidad por completo. Para ambos, el Buen Vivir tiene un carácter plural y de equilibrio, permitiendobeneficiarsede un potencial tanto emancipador como generador de una capacidad dialógica con otros modelos culturales.Vanhulst y Belingseñalan que para que exista tal dialogo de saberes la propuesta del Buen Vivir debe superar los dualismos constitutivos de las formas dominantes de la modernidad, entre ellos el clásico arquetipo del universalismo/ particularismo en el análisis de las relaciones interculturales. Un arquetipo de opuestos donde existe la pretensión de tener un solo modelo cultural y político hegemónico y homogenizante o una posturarelativista postmoderna e impenetrable que no abonan a crear puentes y ceder terreno. El superar el binomio del universalismo/particularismo se enmarca en la necesidad de cuestionar el proyecto desarrollista imperante ante los riesgos ecológicos inminentes en el mundo.Para ello, Vanhulst y Belingconvocan el pensamiento y propuestas de Estermann, Sousa Santos, Rehbeiny otros sobre la necesidad de generar un dialogo de saberes con otros discursos constituyentes del desarrollo sostenible. Dialogo que pretende celebrarse desde un terreno común donde diferentes experiencias de mundo, prácticas, discursos, racionalidades y epistemologías puedan converger, y donde los pueblos puedan ejercer la capacidad que desde siempre se han ejercido; identificar aquello que es útil y desechar lo inútil.

Ello se relaciona con la sociología de las ausencias propuestas por Sousa Santos (2004) como aquellas que el pensamiento hegemónico descarta como inviables, indeseables o impensables y que no pertenecen al repertorio cultural existente. Si el pensamiento hegemónico de la modernidad también tiene los mecanismos y necesidad de descartar y realizar au- 
sencias, para que exista una interfaz libre de hegemonías en todos los sentidos, se requiere que los pensamientos y proyectos alternos, como los de los Buenos Vivires, tengan también esa capacidad y necesidad de seleccionar historias, experiencias, prácticas y capacidad de desechar y realizar ausencias. ${ }^{5}$

La pregunta crucial es: ¿cómo lograr un dialogo de saberes en un terreno esencialmente disparejo caracterizado por relaciones asimétricas entre diferentes cosmologías? Como bien dice Blaser (2012; 17), el problema es cómo lograr una traducción sin el usual intento de forzar, reducir y encuadrar otras realidades dentro de una sola ontología; la moderna. ¿Cómo escapar el régimen moderno que administra lo que es verdad y lo que no sin caer en las provocaciones y trampas de los cánones de la misma Euromodernidad que reproduce dominación y subalternización de conocimientos, sujetos y relaciones? La respuesta podría apuntar, precisamente en la capacidad que deben tener los pueblos de seleccionar, excluirse, retirarse como condición medular para lograr un diálogo de saberes en condiciones de simetría. Tal vez nunca se pueda tener un lugar libre de relaciones de poder en un interfaz de realidades complejas, pero si se pueden tener los mecanismos de asimilar, comprender y rechazar aquello que entra en la conversación. Es decir, que partes constitutivas de los pueblos con otras historias son las que se seleccionan en el dialogo ontológico.

La propuesta de considerar los márgenes como facilitadores del diálogo de saberes, corre en la misma línea que el argumento presentado por Chakrabarty (2007) quien en su libro Provincializing Europa: El pensamiento postcolonial y diferencia histórica ha propuesto una solución convincente al dilema del dominio eurocéntrico. Chakrabarty menciona que no debemos borrar Europa y sustituir su centro con otro; en vez deberíamos apuntar a considerar diversos centros moviendo Europa lejos de su lugar privilegiado de poder hegemónico y provincializándola. De esa manera, otras ontologías, experiencias, forma de vida, sistemas culturales de significados y comprensiones de Buen Vivir se pueden considerar en posiciones horizontales, sin descuidar ninguna diversidad, en lugar de estar en una posición de dominación sobre otra. Asimismo, se argumenta que para mover la hegemonía de la Euro-modernidad -y su reflejo en la figura del Estadohacia un lado, se tiene que considerar igualmente otros centros de conocimiento, otras ontologías. Si bien se parte de que se deben hacer visibles al análisis y escrutinio los entramados de poder que son tejidos por los usuales supuestos existentes de la Euro-modernidad, tejidos que tienden a cubrir e invisibilizar formas alternas de realidades y mundos y por ende, de imponer un único régimen de verdad. También se debe considerar que para resolver los problemas complejos de un mundo multi-ontologicamente globalizado se necesitan de conocimientos y experiencias provenientes de muchas matrices históricas, aun incluidos los de la modernidad.

Esta propuesta hace eco también en el argumento esbozado por los autores de la decolonialidadcuando hablan del pensamiento de frontera, como el ejercicio intelectual de pensar fuera de los supuestos de la modernidad para crear lo que Mignolo (2000; 11-14) llama gnosis o conocimiento 
concebido desde una perspectiva subalterna desde más allá de las fronteras del sistema moderno/colonial para poner en primer plano la creatividad de los conocimientos sumergidos y para contraatacar la narrativa hegemónica del pensamiento occidental.

Elpensamiento de frontera concibe la diversidad como un proyecto de cosmopolitianismo global, entendido este como una reacción critica a la imposición global de un proyecto económico, político y cultural, o bienentendido como una globalización desde abajo (Mignolo 2000a).

Otras sugerencias sobre cómo articular el dialogo en los márgenes provienen de mismoMario Blaser (2010), quien hace referencia no a un pensamiento de frontera sino a un diálogo de frontera. Si bien ambos conceptos implica la posibilidad de lograr un balance en la forma como se produce y interactúan prácticas de conocimiento tanto de la Euro-modernidad como lo alterno a ella, lo no-moderno, la diferencia entre ambos radica en que el pensamiento de frontera se encuentra ya situado en un locus de frontera, mientras que la propuesta de Blaser, implica un primer momento de desplazamiento por parte de la Euro-modernidad hacia ese locus de frontera para que pueda florecer el pensamiento de frontera que señala Mignolo. (Blaser 2010:22). En otras palabras, para que exista diálogo primero tiene que haberun reconocimiento y deslazamiento por parte de la modernidad en su pretensión de ocupar una posición superior entre los sistemas de saberes que transforma toda diferencia en una diferencia colonial.

En este sentido, la propuesta de los márgenes que anuncio en el presente artículo, se entendería como un instrumento hermenéutico donde, además de incorporar una dimensión procesual donde se transite de una necesaria diferenciación radical hacia una plataforma que conlleve al diálogo de saberes, también se esté generando un espacio anti-hegemónico. En los márgenes no solo se existe reapropiación, adaptación y consolidación dinámica de conocimientos y epistemes, también existe política y lucha sobre las condiciones a discutir y construir. Creo que tal posibilidad antihegemónica puede ocurrir sólo en los márgenes, nitotalmente adentro ni totalmente afuera del edificio, sino en complementariedad y en diálogo.

\section{Conclusión}

A lo largo de este artículo se han desarrollado tres puntos esenciales. Primero, la necesidad de establecer que existe una gran variedad de proyectos y discursos asociados al Buen Vivir, y por ende, reconocer el carácter plural del mismo anunciando que existen Buenos Vivires y no un solo y acabado proyecto o discurso del Buen Vivir. La diversidad de tales posturas sobre el Buen Vivir obedece a los numerosos contextos donde proyectos indígenasse hacen presentes desde diversos escenarios y respondiendo a intereses políticos relacionados con la emergencia de nuevas formas de participación y lucha que se han desarrollado en Latinoamérica en las últimas décadas. 
Segundo, argumento que los Buenos Vivires, es decir los proyectos políticos surgidos desde los pueblos con historias diferentes a la modernidad y que enarbolan formas alternas de convivencia y desarrollo, son generalmente analizados y concebidos al margen de ésta. En este aspecto yo postulo que los márgenes no deben ser solo entendidos como lugares periféricos del Estado y/o del pensamiento de la modernidad, sino más bien considerar su posición pluricéntricahaciendo visibles los otros centros políticamente diferenciados ajenos al Estado, al mercado global y otros instancias de la modernidad. Al respecto señalo aspectos del modo de sostenimiento y de relación con la sociedad dominante del pueblo Rarámuri en México y Mapuche en Chile, como ejemplos de articulación política en los márgenes. Es decir, ocupando espacios estatales al demandar derechos igualitarios y ciudadanía diferenciada, y en ocasiones ocupando espacios fuera de los principios del mercado y esferas estatales fortificando su capacidad autogestiva como poseedores de proyectos políticos alternos. Es en este balance de fuerzas, en esa tensión no ausente de conflictos y contradicciones, donde hace sentido conceptualizar los proyectos del Buen Vivir.

Por último, el artículo argumenta como esta fluctuación entre ambas fuerzas hace eco con diversas posturas señaladas por autores de la decolonialidad y del giro ontológico. En esta sección discuto la posibilidad expuesta por Quijano de entender la posición del Buen Vivir desde la perspectivadecolonialde ser un proyecto totalmente ajeno a la modernidad con su propio horizonte epistemológico. En este punto propongo incorporar un sentido procesal en tres momentos; aceptar que un proyecto político que pretende romper con tantas supuestos de la modernidad debe ser considerado el otro radical como primer momento, para pasar a un segundo momento donde habiendo definido su radicalidad, se pueda generar un diálogo de saberes entre posturas ontológicas que reconozca el mismo carácter plural y holístico del Buen Vivir. Como tercer momento argumento, incorporo la necesidad de tener mecanismos dentro de los proyectos políticos alternos que permitan seleccionar aquellos elementos que los pueblos desean incorporar al dialogo para poder asentir y también disentir de los proyectos mayoritarios o impuestos para poder contraatacar relaciones asimétricas persistentes.

La propuesta de entender los diferentes proyectos del Buen Vivir desde los márgenes, esbozada aquí de manera muy somera y preliminar,implica un enfoque que vaya más allá del Estado como monopolio estructurante de verdades y realidades para incluir la existencia de otras comunidades política-ontológicas, digamos el centro de la comunidad Rarámuri o Mapuche, que desafíe prácticas colonialistas y hegemónicas. Entendido así, los diferentes proyectos políticos del Buen Vivir requieren lo que movimientos y foros sociales han expresado en múltiples voces: que otro mundo sea posible, un mundo donde quepan muchos mundos. Más aun, esta mutua existencia, no requiere negar o eliminar necesariamente todo el aparato de la Euro-modernidad, sino más bien seguir la lógica de desechar lo perverso y recuperar lo útil. 
La posibilidad de los Buenos Vivires en los márgenes está basada, en que la naturaleza de este diálogo sea efectivamente una necesidad de lo alterno en la búsqueda de lo posible, tanto como un requerimiento de la modernidad en su búsqueda por la legitimidad. Como dice Boaventura de Sousa Santos (2002) el mundo afronta problemas para los cuales no hay suficientes soluciones modernas. Es considerando ese contexto de mutua y común necesidad donde se puede lograr un diálogo.Por lo tanto, el dialogo de saberes aboga por nuevas formas de comprensión del mundo que están emergiendo. Formas que están en fluctuación entre mundos performativos para buscar oportunidades y alejarse de dominaciones, favorecerse de derechos reconocidos por los Estados, y replegarse para constituir autogestiones. De igual manera el proyecto de los márgenes deberá distanciarse de cualquier práctica que pretenda incluir "al otro" reduciendo su diferencia ontológica a la comprensión moderna dominante, y evitar caer así de nuevo en máscaras multiculturales y fachadas discursivas que sostengan la imperante correlación de dominación y donde se vuelva una crítica de lo eurocéntrico desde el eurocentrismo.

Este proyecto del Buen Vivir en los márgenes tendrá sentido en la medida en que los propios Estados generen esfuerzos reales de integración de múltiples concepciones ontológicas de la naturaleza y la cultura. Tendrá sentido, también en la medida en que el poder de autogestión y organización local de colectivos y comunidades se alejen de las lógicas del mercado, herramientas de participación política usuales, y de los cánones del proyecto de la Euro-modernidad. Con ello se podrá fortalecer la capacidad de los pueblos por fluctuar y perpetuarse no solo en diversidad sino entre los diversos mundosal cual se refería Simón Yampara (2011).Para ello habrá que hacer lo que hacen los Rarámuri; sembrar maíz en diferentes lugares, quizá en un lugar no se dará, pero en otros seguramente sí. 


\section{Notas}

${ }^{1}$ Agradezco el apoyo institucional del Centro Interdisciplinario de Estudios Interculturales e Indígenas del Fondo de Financiamiento de Centros de Excelencia en Investigación (FONDAP) dependiente de la Comisión Nacional de Investigación Científica y Tecnológica (CONICYT), del Ministerio de Educación de Chile.

${ }^{2}$ Para propósitos de este artículo asumo como equivalentes el proyecto de la modernidad a la Euro modernidad y utilizo dichas categorías apelando a su utilidad para un análisis discursivo como el pretendido aquí. Ver Blaser (2009) para una interesante discusión sobre si e existen diferentes modernidades, ontologías modernas o no modernas o si bien no existe como tal la modernidad.

${ }^{3}$ ] La iniciativa Transiciones a las alternativas al desarrollo propuesta por el Centro Latino Americano de Ecología Social (CLAES) es un ejemplo paralelo desde la política pública sobe los que llamo los márgenes. Dicha iniciativa parte de la necesidad de generar modificaciones a las prioridades de los modelos económicos extractivistas imperantes considerando sus impactos sociales y ambientales, y por ende pretende introducir transformaciones radicales en procesos productivos. Ello implica abandonar supuestos profundamente arraigados tales como la necesidad de crecimiento económico constante, o prácticas de alto consumo de materia y energía, pero también beneficiándose de la introducción de nuevas tecnologías y conocimientos. Ver: http:// transiciones.org

${ }^{4}$ Característica de la euro-modernidad es que las diferencias entre los diferentes mundos u ontologías son reducidos a diferencias culturales, es decir un mundo donde existen una multiplicidad de culturas, y no una multiplicidad de mundos (Escobar 2012, Viveiros de Castro 2003). La ironía es que las demandas de reivindicación de los derechos indígenas parten del supuesto de que para ser exigidos y defendidos tienen que ser asumidos como culturas diferenciadas primordialmente, y solo así el multiculturalismo neoliberal puede existir. Si se consideran proyectos políticos alternos, fuera del estado, fuera de sus reglas, entonces tales proyectos corren el riesgo de ser descartados, suprimidos o invisibilizados.

${ }^{5}$ Respecto a la posibilidad anti-hegemónica de los márgenes, se puede buscar inspiración en múltiples instancias. La filosofía deleuziana, por ejemplo, establece una línea de fuga, un intento de liberar y desterritorializar el pensamiento, pero como todas las líneas de fuga minoritarias deben cuestionar lo mayoritario, pero sin sustituirlo; las minorías no deben imponerse, porque entonces se hacen mayoritarias y reproducen aquello que dicen atacar. 


\section{Bibliografía}

Acosta, A. Entre el quiebre y la realidad: Constitución 2008, AbyaYala, Quito.

Idem (2010), El buen vivir en el camino del post-desarrollo. Una lectura desde la constitution de Montecristi. PolicyPaper 9.Quito: Friedrich-EbertStiftung.

Acosta, A\&Gudynas, E. (2011) "El buen vivir o la disolución de la idea del progreso", en Rojas, M (Ed.), La Medición Del Progreso y El Bienestar. Propuestas Desde América Latina, Foro Consultivo Científico y Tecnológico de México, México DF.

Blaser, M.(2009), “Political Ontology”, Cultural Studies, 23 (5), 873-896.

Ídem (2010), Storytelling Globalization from the Chaco and Beyond. Duke University Press, Durham, NC.

Ídem (2014), “Ontology and indigeneity: on the political ontology of heterogeneous assemblages”. Cultural Geographies 21:49-58.

Confederación Mapuce de Neuquén (2010) “Propuesta para un Kvme Felen Mapuce”, Equipo Interdisciplinar e Intercultural del Proyecto. Gráfica Althabe. Newken, PuelMapu.

Clastres, P.(1978), La sociedadContra el Estado. Monte Avila Editores, Barcelona.

Chakrabarty D. (2007), Provincializing Europe: Postcolonial Thought and Historical Difference. Princeton University Press, Princeton

De Sousa Santos, B. (2002), Towards a New Legal Common Sense. Butterworth, Londres.

Idem(2004), The World Social Forum: a users manual. University of Wisconsin, Madison.

Escobar, A.(2012), “Cultura y diferencia: la ontología política del campo de Cultura y Desarrollo”. Wale’keru. Revista de investigación en Cultura y Desarrollo 2, p. 8-29.

Gudynas, E (2011), “Buen Vivir: germinando alternativas al desarrollo”. América Latina en Movimiento No 462: 1-20, 2011. ALAI (Agencia Latino Americana Información), Quito

Loera-Gonzalez, Juan (2013), Conflicting Paths to Wellbeing: Raramuri and Mestizo inter-ethnic relations in Northern Mexico. Tesis doctoral en Estudios del Desarrollo, Universidadde Sussex. 
Idem (2014a), Wellbeing in the Margins: Notes on a New Conceptual Cartography, Alternautas (Re)Searching Development: The AbyaYala Chapter, Disponibleen: http://www.alternautas.net/blog/2014/8/11/ wellbeing-in-the-margins-notes-on-a-new-conceptual-cartography

Ídem (2014b), Buen Vivir, bienestar y ontologías en disputa. Ponencia presentada en el Seminario Políticas de la Naturaleza: Saberes en Disputa y Espacios Vividos, Pontificia Universidad Católica de Chile, 11 y 12 de Diciembre, Disponible en: https://www.academia.edu/10056109/ Buen_Vivir_bienestar_y_ontolog\%C3\%ADas_en_disputa

Loncon, E. (2014), La larga y desafiante tarea de la descolonización lingüística en Chile. Centro de Documentacion Mapuche ÑukeMapu. Disponible en: http://mapuexpress.org/2014/12/15/la-larga-y-desafiante-tarea-dela-descolonizacion-linguistica-en-chile-refundacion-dela\#sthash.egb3vfQj.dpbs

Mignolo, W. (2000), Local Histories/Global Designs: Coloniality, Subaltern Knowledges and Border Thinking. Princeton University Press, Princeton.

Idem(2000), “The many faces of cosmo-polis: Border thinking and critical cosmopolitanism”, Public Culture, 12(3), p.712-748.

Nozick, R. (1974), Anarchy, State and Utopia, Basic Books, Nueva York

Postero, N. (2006), Now We Are Citizens: Indigenous Politics in Postmulticultural Bolivia, Stanford: Stanford Universiy Press.

Povinelli, E. (2011), Economies of Abandonment: Social Belonging and Endurance in Late Liberalism. DukeUniversityPress.

Prada, R. (2008), Subversiones indígenas. Muela del diablo Editores, La Paz.

Quijano, A. (2012), “Buen vivir: entre el “desarrollo” y la des/colonialidad del poder”, Viento Sur, Número 122.

Radcliffe, S. (2011), “Development for a postneoliberal era? SumakKawsay, living well and the limits to decolonization in Ecuador. Geoforum 43, 240249.

Vanhulst, J. \&Beling, A. E (2013), “El Buen Vivir: una utopía latinoamericana en el campo discursivo global de la sustentabilidad”, Polis No. 36, Revista Latinoamericana, Santiago.

Viveiros de Castro, E. (2003), "Perspectivismo y multinaturalismo en la AméricaIndígena,” en: Chaparro Amaya, Adolfo, Christian Schumacher (eds.), Racionalidad ydiscurso mítico, Centro Editorial Universidad del Rosario, ICANH, p. 191-243, Bogotá. 
Idem (2013a), “El Buen Vivir: la irrupción de América Latina en el campo gravitacional del desarrollo sostenible”, REVIBEC -Revista Iberoamericana de Economía Ecológica, Vol. 21 Diciembre, p. 1-14

Yampara, S. (2011), “Cosmovivencia Andina. Vivir y convivir en armonía integral - Suma Qamaña”, Bolivian Studies Journal /Revista de Estudios Bolivianos, 18.

Young, I.M. (1990), Justice and the Politics of Difference. Princeton University Press, Princeton.

Recibido: 19.03.2015

Aceptado: 10.04.2015 